\title{
Degree-ordered percolation on hierarchical scale-free network
}

\author{
Hyun Keun Lee, ${ }^{1,2}$ Pyoung-Seop Shim, ${ }^{3}$ and Jae Dong Noh ${ }^{3,2}$ \\ ${ }^{1}$ Department of Physics and Astronomy, Seoul National University, Seoul 151-747, Korea \\ ${ }^{2}$ School of Physics, Korea Institute for Advanced Study, Seoul 130-722, Korea \\ ${ }^{3}$ Department of Physics, University of Seoul, Seoul 130-743, Korea
}

(Dated: June 19, 2018)

\begin{abstract}
We investigate the critical phenomena of the degree-ordered percolation (DOP) model on the hierarchical $(u, v)$ flower network. Using the renormalization-group like procedure, we derive the recursion relations for the percolating probability and the percolation order parameter, from which the percolation threshold and the critical exponents are obtained. When $u \neq 1$, the DOP critical behavior turns out to be identical to that of the bond percolation with a shifted nonzero percolation threshold. When $u=1$, the DOP and the bond percolation have the same vanishing percolation threshold but the critical behaviors are different. Implication to an epidemic spreading phenomenon is discussed.
\end{abstract}

PACS numbers: 89.75.Hc, 05.70.Fh, 64.60.aq

\section{INTRODUCTION}

Percolation refers to the emergence of a giant cluster during a process where nodes or links are being added. Since a global connectivity is one of the key factors for a proper function of a network, the percolation transition has been received much attention in various contexts. This raises the question regarding the nature of the percolation transition in different applications. The nature depends on whether nodes or links are added in a random or strategic way [1 [5], whether underlying networks are correlated or uncorrelated [6-8], and whether they are static or growing [9, 10].

One of the applications where the percolation plays an important role is the susceptible-infected-removed (SIR) model on complex networks [1]. The SIR model describes an epidemic spreading among individuals which are in a susceptible, infected, or removed state. An infected individual infects a neighboring susceptible one or recovers acquiring immunity. Suppose that one individual is infected while the others are in the susceptible state initially. Then, after a transient period, the system ends up with a state consisting of susceptible and removed individuals. The cluster of removed individuals thus obtained is equivalent to a bond percolation cluster 11]. Hence the epidemic transition is characterized by the percolation transition.

In this paper, we investigate the percolation threshold and the critical behavior of a degree-ordered percolation (DOP) in which nodes are occupied in the descending order of their degree. Higher degree nodes are occupied first. The DOP was introduced in Ref. [12] in order to resolve a controversy concerning the epidemic threshold for an infection rate $\lambda$ of the susceptible-infectedsusceptible (SIS) model. In contrast to the SIR model, infected individuals become susceptible again after recovering. Thus, the SIS model does not correspond to an ordinary percolation model.

The SIS model was studied in the framework of the heterogeneous mean-field (HMF) theory in which node connectivity was treated in an annealed way [13. It predicts a threshold $\lambda_{c}^{\mathrm{HMF}}$ as a function of cumulants of the degree, which may be zero or nonzero depending on the degree distribution. Later on, a more refined quenched mean-field (QMF) theory was proposed, in which node connectivity was treated in a quenched way $14-16]$. The QMF yields that the density $\rho$ of infected nodes becomes nonzero at $\lambda_{c}^{\mathrm{QMF}} \lesssim 1 / \sqrt{k_{\max }}$ where $k_{\max }$ is the highest degree among all nodes. It leads researchers to conclude that the epidemic threshold vanishes in any networks where the maximum degree diverges in the infinite-size limit [15, 16].

This conclusion might be true if $\rho$ would remain finite in the infinite-size limit for $\lambda>\lambda_{c}^{\mathrm{QMF}}$. However, it was found that the infection of the QMF theory can be localized around the hub with the maximum degree for a finite $\lambda$ larger than $\lambda_{c}^{\text {QMF }}$ [17]. The localization property was more elaborated in Ref. [12]. As $\lambda$ increases, more localized infections appear around nodes whose degree $k$ satisfies $k \gtrsim 1 / \lambda^{2}$. Localized infections are finite-sized, hence decay after a transient period. The true epidemic transition will take place when the localized infections percolate at $\lambda_{c}>\lambda_{c}^{\mathrm{QMF}}$. Note that localized infections appear successively around nodes in the degree-descending order. Thus, the DOP can provide a valuable information on the epidemic threshold. It was claimed that a nonzero DOP threshold implies a nonzero epidemic threshold [12], which was confirmed numerically in a model network. The controversy is still under debate [18, 19].

A complex network is vulnerable to a targeted attack to higher degree nodes [3]. The DOP deals with the opposite situation where higher degree nodes are preferred. It is an interesting question whether the preference can make the percolation threshold vanish in complex networks. Furthermore, considering the relevance to the SIS model, it is important to establish firmly the percolation threshold and the critical property of the DOP in complex networks whose maximum degree diverges in the infinite size limit. For this reason, we investigate the DOP in the $(u, v)$ flower networks [20]. The $(u, v)$ flow- 
a)
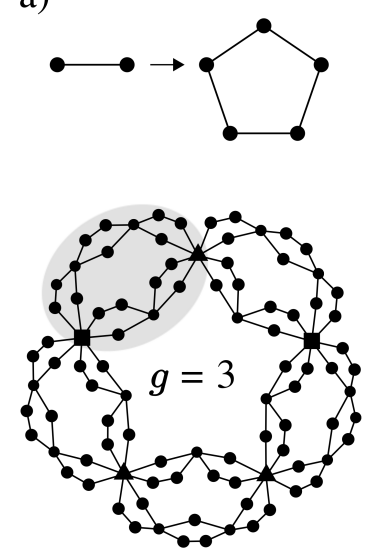

b)
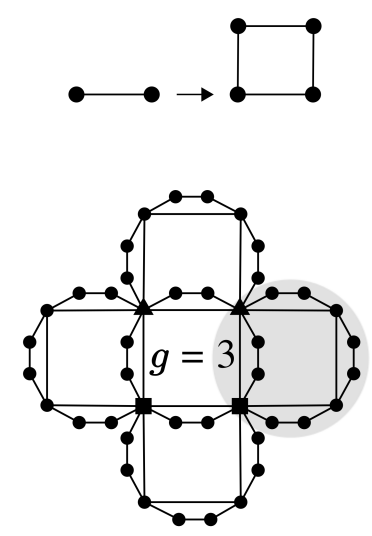

FIG. 1. Iteration rules and and configurations of $G_{3}$ for the $(2,3)$ flower in (a) and $(1,3)$ flower in (b). The roots and the hubs are denoted by square and triangular symbols, respectively. The shaded area represents one of the descendents of $G_{3}$.

ers have a hierarchical structure, which allows an analytic approach. It is best suited for our purpose.

This paper is organized as follows: In Sec. III, we apply the DOP to the hierarchical $(u, v)$ flowers and find a recursion relation for the percolating probability. It allows us to find the percolation threshold and the finite-size scaling exponent exactly. In Sec. III] we derive a set of recursion relations for the percolation order parameters, and obtain the order parameter exponent. We summarize the paper with discussion in Sec. IV

\section{PERCOLATION THRESHOLD}

The $(u, v)$ flower is a model for a scale-free network with hierarchical structure [20]. It is generated iteratively starting from a zeroth generation $G_{0}$ consisting of two nodes and a link connecting them. Given a gth generation $G_{g}, G_{g+1}$ is obtained by replacing each link with two linear chains, one of which consists of $u$ links and $(u-1)$ nodes and the other of which consists of $v$ links and $(v-1)$ nodes [see Fig. 1. Among others, the initial nodes present in $G_{0}$ and $G_{1}$ play an important role in characterizing the percolation. The two nodes of $G_{0}$ will be referred to as roots, and $w \equiv u+v$ nodes of $G_{1}$ as hubs. A root is also a hub.

Alternatively, $G_{g+1}$ can be understood as the $w$ copies of $G_{g}$ that are arranged along a ring with the roots of adjacent $G_{g}$ s being joined. Hereafter, $G_{g}$ embedded in $G_{g+1}$ is called a descendent, and embedding $G_{g+1}$ is called an ascendent. The roots of adjacent descendents are merged to become a hub of the ascendent. Without loss of generality, it is assumed that $u \leq v$.

Summarized below are the structural properties of the $(u, v)$ flower of generation $g$ [20]: (i) The total number

of nodes is given by $N_{g}=\frac{w-2}{w-1} w^{g}+\frac{w}{w-1}$, which is obtained from the recursion relation $N_{g}=w\left(N_{g-1}-1\right)$ with $N_{0}=2$. (ii) The degree of nodes takes on a value among $\left\{2^{1}, \cdots, 2^{g}\right\}$, and the number of nodes having degree $k=2^{l}(l=1, \cdots, g)$ is given by $n_{l}=(w-2) w^{g-l}$ for $l<g$ and $n_{g}=w$. (iii) The cumulative degree distribution is defined as $P_{\geq}(k) \equiv \sum_{2^{l} \geq k} n_{l} / N_{g}$. It decays as $P_{\geq}(k) \sim k^{1-\gamma}$ with $\gamma=1+\ln w / \ln 2$ in the large $k$ limit. (iv) The $(u, v)$ flower is a small-world network with the diameter scaling as $D_{g} \sim \ln N_{g}$ for $u=1$ while it is a fractal network with $D_{g} \sim N_{g}^{\ln u / \ln w}$ for $u>1$.

We describe the DOP on the $(u, v)$ flower. Initially all the nodes are unoccupied or empty. Then, one selects a node with the highest degree among empty nodes and marks it as an occupied node. In case there are multiple candidates, one of them is selected randomly. This procedure is repeated until the fraction of occupied nodes reaches a prescribed value $p$.

We are interested in the percolating probability denoted by $\Pi=\Pi_{g}(p)$. It is defined as the probability that the two roots of $G_{g}$ are connected via occupied nodes. One needs to treat the cases with $u=1$ and $u>1$ separately. When $u=1$, the roots are connected via a single link in all generations. Thus, the system is percolating if the two roots are occupied. This implies that $\Pi=1$ for $p \geq n_{g} / N_{g} \sim w^{-g}$, so the percolation threshold vanishes in the infinite-size limit.

When $u>1$, any path connecting the two roots includes nodes with the minimum degree $k=2$ [see Fig. 1]. Consequently, all nodes with $k>2$ should be occupied necessarily for percolation. Remaining nodes have the same degree $k=2$, so they are occupied randomly. Hence, the DOP in the $(u, v)$ flowers with $u>1$ is equivalent to the ordinary random node percolation starting from the correlated initial condition in which all nodes with $k>2$ are occupied. Let $r$ be the occupation probability of nodes with $k=2$. It is related to the overall fraction $p$ of occupied nodes through

$$
p=p_{0}+\frac{n_{1}}{N_{g}} r
$$

with $p_{0}=1-n_{1} / N_{g}$. In the infinite-size limit $(g \rightarrow \infty)$, Eq. (1) becomes

$$
p=\frac{1+(w-1) r}{w} .
$$

The percolating probability is regarded as a function of $p$ or $r$.

The hierarchical structure allows us to derive a recursion relation for the percolating probability. Any path connecting the two roots of $G_{g}$ necessarily passes through intermediate $(u-1)$ hubs in one direction or $(v-1)$ hubs in the other direction. Note that the hubs of an ascendent $\left(G_{g}\right)$ is the roots of the descendents $\left(G_{g-1}\right)$. Hence, $G_{g}$ is percolated only when $u$ descendents or $v$ descendents are percolated simultaneously. The roots of all descendents are always occupied with $k>2$ and the 


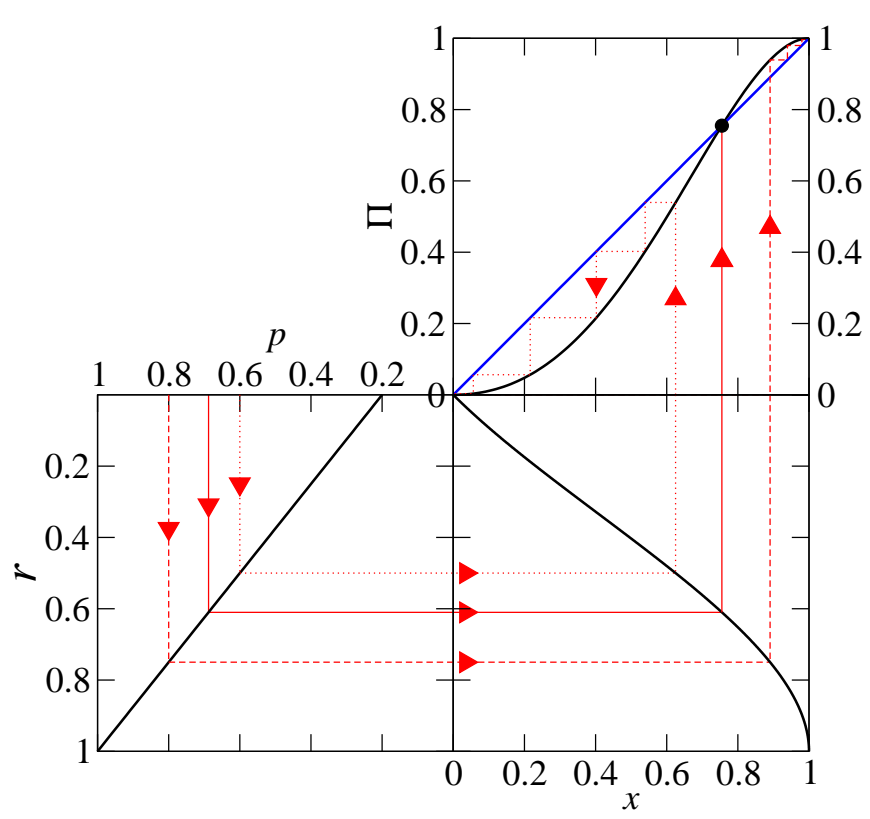

FIG. 2. (Color online) Graphical representation of the recursion relation for $\Pi_{g}$ at $(u, v)=(2,3)$.

descendents are independent and statistically identical. This yields that

$$
\Pi_{g}(r)=f\left(\Pi_{g-1}(r)\right)
$$

with

$$
f(x) \equiv 1-\left(1-x^{u}\right)\left(1-x^{v}\right)
$$

with $g>1$. One need to be cautious in applying the recursion relation to the case with $g=2$. In the recursion relation, $\Pi_{1}(r)$ should be interpreted as the percolating probability of a descendent of $G_{2}$. The roots of a descendent is occupied since their degree is $k=4$. Thus, we obtain

$$
\Pi_{1}(r)=h(r)
$$

with

$$
h(x) \equiv 1-\left(1-x^{u-1}\right)\left(1-x^{v-1}\right) .
$$

Note that the actual percolating probability of an isolated $G_{1}$, not a descendent of $G_{2}$, is given by $r^{2} h(r)$.

The recursion relation is represented graphically in Fig. 2 To a given value of $p$, one obtains $r$ from Eq. (2) and $\Pi_{1}(r)$ from Eq. (5). Inserting this value into Eq. (3) iteratively, one obtains the percolating probability $\Pi_{g}$ for all $g>1$. Figure 3 shows the percolating probability obtained from the recursion relation in $(2,3)$ flowers. Also shown are Monte Carlo simulation data for comparison.

The percolating probability converges to 0 (1) in the $g \rightarrow \infty$ limit when $r$ is less (greater) than a threshold value $r_{c}$. It takes a fixed point value $\Pi_{c}=x_{c}$ at the

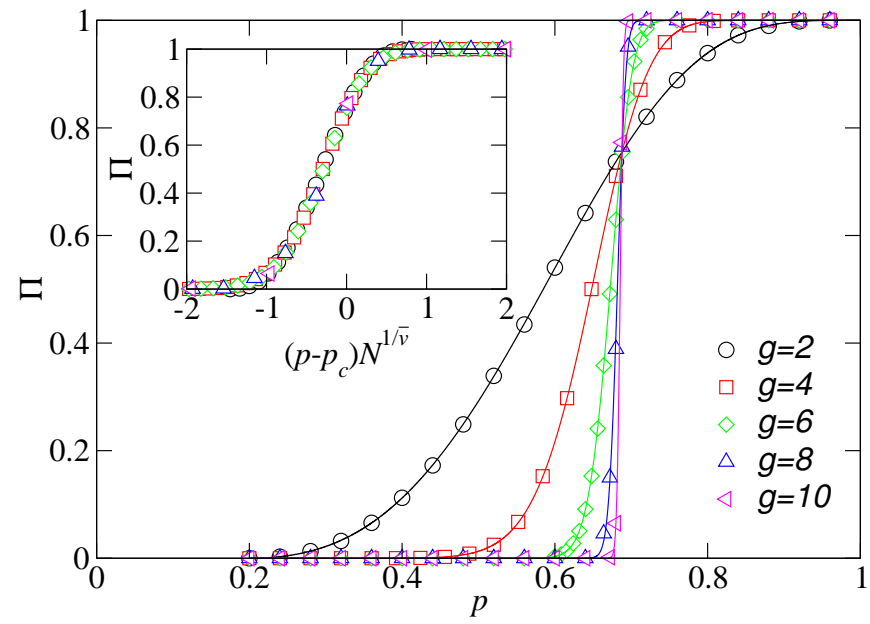

FIG. 3. (Color online) $\Pi_{g}(p)$ from the recursion relation (solid lines) and from Monte Carlo simulations (symbols) in $(2,3)$ flowers. Inset shows the finite-size-scaling analysis of the percolating probability.

TABLE I. Percolation thresholds and critical exponents for the DOP on $(u, v)$ flowers.

\begin{tabular}{cccccc}
\hline \hline$(u, v)$ & $\gamma$ & $p_{c}$ & $\Pi_{c}$ & $\bar{\nu}$ & $\beta$ \\
\hline$(2,2)$ & 3.000 & 0.536 & 0.618 & 3.271 & 0.165 \\
$(2,3)$ & 3.322 & 0.688 & 0.755 & 3.444 & 0.112 \\
$(3,3)$ & 3.585 & 0.818 & 0.848 & 3.448 & 0.053 \\
$(2,4)$ & 3.585 & 0.762 & 0.819 & 3.696 & 0.103 \\
$(3,4)$ & 3.807 & 0.869 & 0.890 & 3.595 & 0.040 \\
$(4,4)$ & 4.000 & 0.909 & 0.921 & 3.682 & 0.025 \\
$(2,5)$ & 3.807 & 0.808 & 0.857 & 3.936 & 0.100 \\
$(3,5)$ & 4.000 & 0.898 & 0.913 & 3.759 & 0.036 \\
$(4,5)$ & 4.170 & 0.929 & 0.938 & 3.804 & 0.020 \\
$(5,5)$ & 4.322 & 0.946 & 0.951 & 3.896 & 0.015 \\
$(2,6)$ & 4.000 & 0.838 & 0.881 & 4.156 & 0.100 \\
$(3,6)$ & 4.170 & 0.915 & 0.929 & 3.919 & 0.034 \\
$(4,6)$ & 4.322 & 0.942 & 0.949 & 3.931 & 0.018 \\
$(5,6)$ & 4.459 & 0.956 & 0.960 & 3.999 & 0.012 \\
$(6,6)$ & 4.585 & 0.964 & 0.967 & 4.084 & 0.010 \\
\hline \hline
\end{tabular}

threshold $r=r_{c}$ or $p=p_{c}=\left(1+(w-1) r_{c}\right) / w$. The threshold is given by

$$
r_{c}=h^{-1}\left(x_{c}\right),
$$

where $x_{c} \neq 0,1$ is the solution of

$$
x_{c}=f\left(x_{c}\right) .
$$

The thresholds at several values of $(u, v)$ are listed in Table I

The percolating probability satisfies the finite-sizescaling (FSS) form [22, 23]

$$
\Pi_{g}(r)=\mathcal{F}\left(\epsilon N_{g}^{1 / \bar{\nu}}\right)
$$


where $\epsilon=p-p_{c}, \bar{\nu}$ is the FSS exponent, and $\mathcal{F}(x)$ is the scaling function. It has the limiting behaviors $\mathcal{F}(x \rightarrow$ $-\infty) \rightarrow 0, \mathcal{F}(0)=x_{c}$, and $\mathcal{F}(x \rightarrow \infty) \rightarrow 1$. Combining Eqs. (3) and (9), we find

$$
\bar{\nu}=\frac{\ln w}{\ln f^{\prime}\left(x_{c}\right)} .
$$

Numerical values of $\bar{\nu}$ are listed in Table 1 The FSS form is tested for the $(2,3)$ flowers in the inset of Fig. 3 .

It is noteworthy that the recursion relation for the percolating probability of the DOP in the $(u, v)$ flowers with $u \neq 1$ is similar to that of the ordinary bond percolation studied in Ref. 21]. The difference lies in the fact that the recursion relation is written in terms of $r$ instead of $p$ and that $\Pi_{1}$ has a different form. The difference only shifts the percolation threshold. The critical behaviors belong to the same universality class. When $u=1$, the DOP and the ordinary bond percolation have the same percolation threshold at $p_{c}=0$. However, they display different critical phenomena as will be shown in the following section.

\section{PERCOLATION ORDER PARAMETER}

In this section, we investigate the critical scaling of the percolation order parameter. It is defined as the mean density of nodes that are connected to any of the hubs.

We first consider the case with $u>1$. Nodes with $k>2$ are all occupied and remaining nodes with $k=2$ are occupied with probability $r$. The order parameter $P_{\infty}=P_{\infty}(g, r)$ is written as

$$
P_{\infty}(g, r)=\frac{1}{N_{g}} \sum_{m=1}^{w} A_{g, m}(r)
$$

where $A_{g, m}(r)$ denotes the mean number of nodes in $G_{g}$ that are connected to exactly $m$ hubs under the condition that the two roots of $G_{g}$ are occupied.

It is useful to introduce $S=S_{g}(r)$, the mean number of nodes in $G_{g}$ that are connected to only a single root. It is related to $A_{g, m}$ through the relation

$$
S_{g}(r)=\sum_{m=1}^{w} \sigma_{m} A_{g, m}(r)
$$

where $\sigma_{m}$ is the probability that one may find only a single root among randomly selected $m$ adjacent hubs. Note there are $w$ different ways in selecting $m$ adjacent hubs except when $m=w$. Enumerating all the possible cases, we obtain that

$$
\sigma_{m}= \begin{cases}\frac{2 m}{w} & (1 \leq m \leq u), \\ \frac{2 u}{w} & (u<m \leq v), \\ \frac{2(w-m)}{w} & (v<m \leq w) .\end{cases}
$$

We also introduce $T=T_{g}(r)$, the mean number of nodes in $G_{g}$ that are connected to both roots. It is given by

$$
T_{g}(r)=\sum_{m=1}^{w} \tau_{m} A_{g, m}(r)
$$

where $\tau_{m}$ is the probability that one may find both roots among randomly selected $m$ adjacent hubs. It is given by

$$
\tau_{m}= \begin{cases}0 & (1 \leq m \leq u) \\ \frac{m-u}{w} & (u<m \leq v) \\ \frac{(2 m-w)}{w} & (v<m \leq w) .\end{cases}
$$

Note that $G_{g+1}$ (an ascendent) consists of $w$ copies of $G_{g}$ (descendents) with their roots being joined. Hence, one finds that $A_{g+1, m}=w\left(X_{g, m}-c_{g, m}\right)$, where $X_{g, m}$ denotes the mean number of nodes in one descendent that are connected to exactly $m$ hubs of the ascendent and $c_{g, m}$ denotes the probability that a hub of the ascendent is connected to $(m-1)$ other hubs. Because each hub of the ascendent is shared by two adjacent descendents, one has to subtract $w c_{g, m}$ from $w X_{g, m}$ in order to compensate for a double counting.

For $m=1$, we obtain that $X_{g, 1}=S_{g} \bar{\Pi}_{g}$, where $\bar{\Pi}_{g} \equiv$ $1-\Pi_{g}$ with the percolating probability $\Pi_{g}$. The factor $S_{g}$ accounts for the number of nodes in a descendent that are connected to one of the roots (or, equivalently, one of the hubs of the ascendent), and the factor $\bar{\Pi}_{g}$ accounts for the probability that such nodes are not connected to any other hubs of the ascendent. A hub of $G_{g+1}$ is not connected to the adjacent hubs with the probability $\bar{\Pi}_{g}^{2}$, which yields $c_{g, 1}=\bar{\Pi}_{g}^{2}$. For general $m$, one can easily find that

$$
A_{g+1, m}=w\left(a_{g, m} S_{g}+b_{g, m} T_{g}-c_{g, m}\right)
$$

where

$$
\begin{gathered}
a_{g, m}=\left\{\begin{array}{lr}
\Pi_{g}^{m-1} \bar{\Pi}_{g} & (1 \leq m<w) \\
\Pi_{g}^{w-1} & (m=w)
\end{array}\right. \\
b_{g, m}=\left\{\begin{array}{lr}
(m-1) \Pi_{g}^{m-2} \bar{\Pi}_{g}^{2} & (1 \leq m<w) \\
(w-1) \Pi_{g}^{w-2} \bar{\Pi}_{g}+\Pi_{g}^{w-1} & (m=w)
\end{array}\right.
\end{gathered}
$$

and

$$
c_{g, m}=\left\{\begin{array}{lr}
m \Pi_{g}^{m-1} \bar{\Pi}_{g}^{2} & (1 \leq m<w) \\
w \Pi_{g}^{w-1} \bar{\Pi}_{g}+\Pi_{g}^{w} & (m=w)
\end{array} .\right.
$$

Once $\left\{A_{1, m}\right\}$ are known, $\left\{A_{g>1, m}\right\}$ are obtained by using Eqs. (12), (14), and (16). The order parameter is then evaluated by using Eq. (11). 


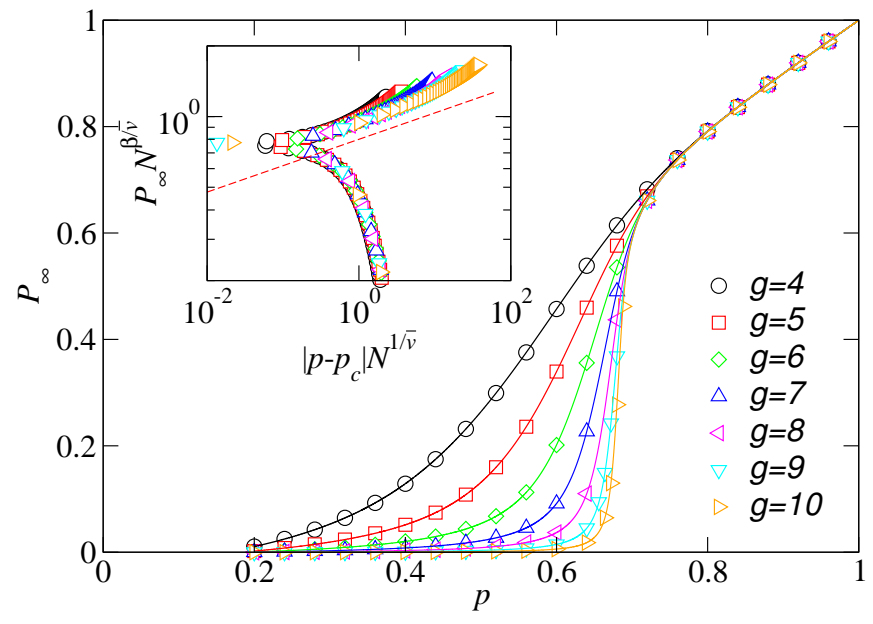

FIG. 4. (Color online) The percolation order parameter $P_{\infty}$ obtained from the recursion relation (solid lines) and Monte Carlo simulations (symbols) in $(2,3)$ flowers. The inset shows the FSS analysis of the order parameter. The slope of the dashed line is $\beta$.

It is more convenient to express the order parameter in terms of $S_{g}$ and $T_{g}$. By using Eq. (16) and $\sum_{m} a_{g, m}=$ $\sum_{m} b_{g, m}=\sum_{m} c_{g, m}=1$, we find that

$$
P_{g+1}=\frac{1}{N_{g+1}} \sum_{m=1}^{g} A_{g+1, m}=\frac{w}{N_{g+1}}\left(S_{g}+T_{g}-1\right) .
$$

Combining Eqs. (12), (14), and (16), one can derive the following recursion relation

$$
\left(\begin{array}{c}
S_{g+1} \\
T_{g+1}
\end{array}\right)=w \mathrm{M}_{g}\left(\begin{array}{c}
S_{g} \\
T_{g}
\end{array}\right)-w\left(\begin{array}{c}
\sum_{m} \sigma_{m} c_{g, m} \\
\sum_{m} \tau_{m} c_{g, m}
\end{array}\right),
$$

where $\mathrm{M}_{g}$ is the $2 \times 2$ matrix given by

$$
\mathbf{M}_{g}=\left(\begin{array}{ll}
\left(\sum_{m} \sigma_{m} a_{g, m}\right) & \left(\sum_{m} \sigma_{m} b_{g, m}\right) \\
\left(\sum_{m} \tau_{m} a_{g, m}\right) & \left(\sum_{m} \tau_{m} b_{g, m}\right)
\end{array}\right) .
$$

The recursion relation is tested numerically for $(2,3)$ flowers. In $G_{1}$ as a descendent of $G_{2}$, two roots are occupied with probability 1 and other hubs are occupied with probability $r$. It is straightforward to obtain that $S_{1}=2(1+2 r)(1-r)^{2}$ and $T_{1}=r\left(3+6 r-4 r^{2}\right)$. Inserting these into Eqs. (20) and (21) iteratively, we obtain the order parameter in all $g$. The numerical results are presented and compared with Monte Carlo simulation results in Fig. 4. Both agree with each other perfectly.

The critical scaling behavior of the order parameter is also obtained by analyzing the recursion relation. The order parameter is expected to follow the FSS form 22]

$$
P_{\infty}(p)=N_{g}^{-\beta / \bar{\nu}} \mathcal{G}\left(\epsilon N^{1 / \bar{\nu}}\right),
$$

where $\epsilon=p-p_{c}$ and the scaling function has the limiting behaviors $\mathcal{G}(x \rightarrow \infty) \sim x^{\beta}$ and $\mathcal{G}(|x| \ll 1) \sim$ const. with the order parameter exponent $\beta$. In the preceding section, we have obtained the critical point $p_{c}$ or $r_{c}$ and the FSS exponent $\bar{\nu}$. Hence, the exponent $\beta$ can be obtained from the FSS behavior of the order parameter at $p=p_{c}$ :

$$
P_{\infty}\left(p_{c}\right) \sim N_{g}^{-\beta / \bar{\nu}} .
$$

In the subcritical phase, the number of nodes connected to the hubs are order of unity with $P_{\infty}\left(p<p_{c}\right) \sim N_{g}^{-1}$. Hence, it is expected that $\beta / \bar{\nu}<1$.

At the critical point with $\beta / \bar{\nu}<1$, we can ignore the last terms in Eqs. (20) and (21) and use the fixed point value $\Pi_{c}$ instead of $\Pi_{g}$. Then, the order parameter scales as

$$
P_{\infty} \sim\left(w \Lambda_{c}\right)^{g} / N_{g} \sim \Lambda_{c}^{g} \sim N_{g}^{\ln \Lambda_{c} / \ln w},
$$

where $\Lambda_{c}$ is the largest eigenvalue of $M_{c}=\left.M_{g}\right|_{\Pi_{g}=\Pi_{c}}$. Comparing Eqs. (24) and (25), we find that

$$
\frac{\beta}{\bar{\nu}}=-\frac{\ln \Lambda_{c}}{\ln w} .
$$

The knowledge of $\bar{\nu}$ and $\beta$ completes the critical behavior of the DOP on the $(u, v)$ flowers.

Consider the $(2,3)$ flower as an example. We have $\Pi_{c} \simeq 0.755$ and $\bar{\nu} \simeq 3.44$ in Table \. The matrix $\mathrm{M}_{c}$ is given by

$$
\mathrm{M}_{c}=\left(\begin{array}{ccccc}
\frac{2}{5} & \frac{4}{5} & \frac{4}{5} & \frac{2}{5} & 0 \\
0 & 0 & \frac{1}{5} & \frac{3}{5} & 1
\end{array}\right)\left(\begin{array}{cc}
\bar{\Pi}_{c} & 0 \\
\Pi_{c} \bar{\Pi}_{c} & \bar{\Pi}_{c}^{2} \\
\Pi_{c}^{2} \bar{\Pi}_{c} & 2 \Pi_{c} \bar{\Pi}_{c}^{2} \\
\Pi_{c}^{3} \bar{\Pi}_{c} & 3 \Pi_{c}^{2} \bar{\Pi}_{c}^{2} \\
\Pi_{c}^{4} & 4 \Pi_{c}^{3} \bar{\Pi}_{c}+\Pi_{c}^{4}
\end{array}\right),
$$

with $\bar{\Pi}_{c}=1-\Pi_{c}$. It has the largest eigenvalue $\Lambda \simeq$ $0.948820 \cdots$, which yields that $\beta / \bar{\nu} \simeq 0.0326426 \cdots$ and $\beta \simeq 0.112424 \cdots$. The inset of Fig. 4 shows that the numerical data indeed follow the FSS scaling form. The critical exponents at several values of $u$ and $v$ are listed in Table \

We add a remark on our recursion relation given in Eqs. (20) and (21) in comparison with that obtained for the random bond percolation in Ref. [21]. The recursion relations in both studies look similar to each other except for the terms involving $c_{g, m}$ in Eq. (21). Those terms account for the double counting of hubs. Such terms are overlooked in Ref. [21]. Fortunately, they contribute to the order parameter as a subleading correction term. Hence, the results for the critical exponents in Ref. 21 are valid. The double counting problem is also noticed in Ref. [24].

Finally, we investigate the nature of the percolation transition in $(u=1, v)$ flowers, where the percolation transition takes place at $p=p_{c}=0$. We describe how the occupied cluster changes its shape as $p$ increases. When $p=P_{>}\left(k=2^{g}\right)$, all the hubs with $k=2^{g}$ are occupied and connected to each other. Suppose that 
$P_{\geq}\left(2^{g}\right)<p \leq P_{\geq}\left(2^{g-1}\right)$ or $p=P_{\geq}\left(2^{g}\right)+s n_{g-1} / N_{g}$ with $0<s \leq 1$. Then, one needs to consider the nodes with $k=2^{g-1}$ additionally. These nodes form linear chains of length $v-1$ connecting two neighboring hubs. Figure 5 illustrates a unit consisting of a pair of hubs (closed symbols) and the linear chain of $(v-1)$ nodes with $k=2^{g-1}$ (open symbols). Those nodes that are occupied and connected to the hubs contribute to the percolation order parameter. When $s=1$, all nodes are connected to each other to form a single cluster.

As $p$ increases further, we have a nested structure of the units. Suppose, in general, that $P_{\geq}\left(2^{l}\right)<p \leq P_{\geq}\left(2^{l-1}\right)$ or $p=P_{\geq}\left(2^{l}\right)+s n_{l-1} / N_{g}$ with a certain $l$ and $0<s \leq 1$. All the nodes with $k \geq 2^{l}$ are occupied with probability 1 and belong to a single cluster. In addition, nodes with $k=2^{l-1}$ are attached to every neighboring pair of nodes of higher degree to form the unit structure in Fig. 5 (see also Fig. (1). Given the occupation probability $s$ of the nodes with $k=2^{g-l}$ (open symbols), the probability $q_{m}$ that $m$ nodes among $v-1$ nodes in the unit are connected to the higher degree nodes (closed symbols) is given by

$$
q_{m}=\left\{\begin{array}{ll}
(m+1) s^{m}(1-s)^{2} & (m<v-2) \\
(m+1) s^{m}(1-s) & (m=v-2) \\
s^{m} & (m=v-1)
\end{array} .\right.
$$

The mean value is given by

$$
q(s)=\sum_{m} m q_{m}=\frac{(v-3) s^{v}-(v-1) s^{v-1}+2 s}{1-s} .
$$

Therefore, the percolation order parameter is given by

$$
P_{\infty}(p)=P_{\geq}\left(k=2^{l}\right)+\frac{q(s)}{(v-1)} \frac{n_{l-1}}{N_{g}},
$$

where $l$ is the smallest integer satisfying $p>P_{\geq}\left(2^{l}\right)$ and $s=\left(p-P_{\geq}\left(2^{l}\right)\right) / P\left(2^{l-1}\right)$.

When $v=2$ or $v=3$, all nodes selected in the degree descending order are connected to each other. Indeed, Eq. (29) yields $q(s)=(v-1) s$ and the percolation order parameter becomes $P_{\infty}(p)=p$. When $v>3, q(s)$ deviates from $(v-1) s$. Consequently, $P_{\infty}(p)$ displays an oscillatory behavior with the period $\Delta p / p \simeq 1 / w$ superimposed over the overall behavior $P_{\infty}(p)=p$. The percolation order parameter $P_{\infty}(p)$ calculated from Monte Carlo simulations is compared with the analytic result in Fig. 6. Both are in perfect agreement with each other. Therefore, we conclude that the DOP on $(u=1, v)$ flowers has a percolation threshold at $p_{c}=0$ and the order parameter exponent is given by $\beta=1$.

\section{SUMMARY AND DISCUSSIONS}

We have introduced the DOP and investigated the critical phenomena in the $(u, v)$ flower networks. The hierarchical structure of the $(u, v)$ flowers allows us to find the

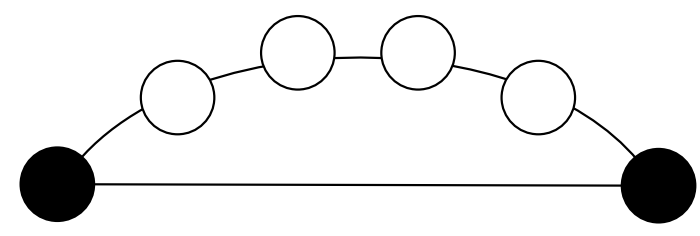

FIG. 5. A diagram illustrating the unit of $(v-1)$ nodes with $k=2^{l}$ (denoted by open symbols) connecting two nodes with $k=2^{l^{\prime}}$ with $l^{\prime}>l$ (denoted by closed symbols).

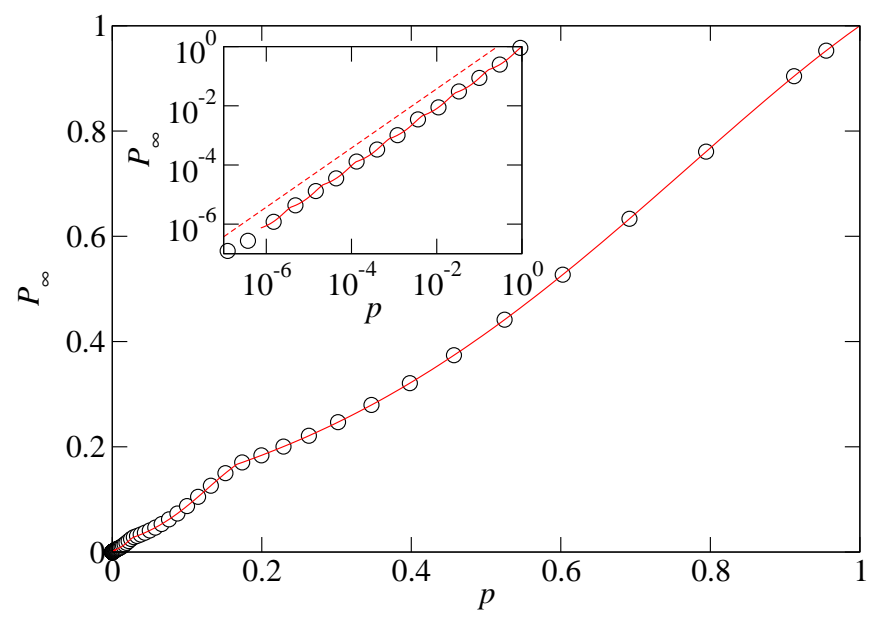

FIG. 6. (Color online) Order parameter obtained from Monte Carlo simulations (symbol) and from Eq. (30) (line) in the $(1,5)$ flower in generation $g=9$. The inset shows the plot in the $\log -\log$ scale. The straight line of slope 1 is a guide to an eye.

percolation threshold and the critical exponents exactly. When $u=1$, the percolation transition takes place at $p=p_{c}=0$ with the order parameter exponent $\beta=1$. When $u \neq 1$, the percolation threshold is nonzero and the critical exponents take the nontrivial values depending on $u$ and $v$. The percolation thresholds and the critical exponents are summarized in Table \.

The ordinary bond percolation on the $(u, v)$ flowers was investigated in Ref. 21]. Comparing the two studies, we find that the DOP and the ordinary bond percolation belong to the same universality class for $u \neq 1$. On the other hand, the critical behaviors are different when $u=1$. The ordinary bond percolation transition is of infinite order with $\beta=\infty$, while the DOP transition is characterized with $\beta=1$. Recently, the ordinary site percolation on $(1,2)$ flowers was studied in Ref. 24]. It was found that the system is critical in the region $0<p<$ 1 and the percolation transition takes place at $p=p_{c}=1$. Thus, when $u=1$, the DOP transition is also distinct from the ordinary site percolation transition.

It is interesting to note that the DOP and the ordinary percolation belong to the same universality class when $u \neq 1$. The result is consistent with the findings of Ref. [4] studying a percolation transition of scale-free 
networks under a targeted attack. Each node $i$ with degree $k_{i}$ fails with a probability $W\left(k_{i}\right) \propto k_{i}^{\alpha}$ with a control parameter $\alpha$. As the fraction of failed nodes varies, the giant cluster of working nodes undergo a percolation transition. When $\alpha=0$, it is equivalent to the ordinary site percolation. For positive values of $\alpha$, the larger degree a node has, the more vulnerable it is, and vice versa. It was found in Ref. [4] that the percolation transitions with negative $\alpha$ belong to the same universality class as the random percolation transition $(\alpha=0)$. The DOP corresponds to the case with $\alpha=-\infty$. It is surprising that the extreme-case percolation displays the same critical phenomena as the random percolation.

In Ref. [12], we proposed that the epidemic threshold of the SIS model on a network can be zero only when the threshold of the DOP on the same network is zero. The epidemic threshold was found to be zero at $u=1$ and nonzero for $u \neq 1$ numerically [12]. The analytic results for the DOP threshold for the $(u, v)$ flowers support the proposal. The DOP in general random scale-free networks is left for future work.

\section{ACKNOWLEDGMENTS}

This work was supported by the Basic Science Research Program through the NRF Grant No. 2013R1A2A2A05006776. This work was also supported by the NRF Grant No. 2010-0015066.
[1] R. Cohen, K. Erez, D. ben-Avraham, and S. Havlin, Phys. Rev. Lett. 85, 4626 (2000).

[2] D.S. Callaway, M.E.J Newman, S.H. Strogatz, and D.J. Watts, Phys. Rev. Lett. 85, 5468 (2000).

[3] R. Albert, H. Jeong, and A.-L. Barabási, Nature 406, 378 (2000).

[4] L.K. Gallos, R. Cohen, P. Argyrakis, A. Bunde, and S. Havlin, Phys. Rev. Lett. 94, 188701 (2005).

[5] H. Hooyberghs, B. Van Schaeybroeck, A.A. Moreira, J.S. Andrade, H.J. Herrmann, and J. O. Indekeu, Phys. Rev. E 81, 011102 (2010).

[6] J.D. Noh, Phys. Rev. E 76, 026116 (2007).

[7] S.-W. Kim and J.D. Noh, J. Korean Phys. Soc. 52, S145 (2008).

[8] A.V. Goltsev, S.N. Dorogovtsev, and J.F.F. Mendes, Phys. Rev. E 78, 051105 (2008).

[9] D.S. Callaway, J.E. Hopcroft, J.M. Kleinberg, M.E.J. Newman, and S.H. Strogatz, Phys. Rev. E 64, 041902 (2001).

[10] J. Kim, P.L. Krapivsky, B. Kahng, and S. Redner, Phys. Rev. E 66, 055101(R) (2002).

[11] M.E.J. Newman, Phys. Rev. E 66, 016128 (2002).

[12] H. K. Lee, P.-S. Shim, and J. D. Noh, Phys. Rev. E 87, 062812 (2013).
[13] R. Pastor-Satorras and A. Vespignani, Phys. Rev. Lett. 86, 3200 (2001); Phys. Rev. E 63, 066117 (2001).

[14] Y. Wang et al., in 22nd International Symposium on Reliable Distributed Systems (SRDS'03), Los Alamitos, CA, USA (IEEE, Piscataway, NJ, 2003), pp. 25-34.

[15] C. Castellano and R. Pastor-Satorras, Phys. Rev. Lett. 105,218701 (2010).

[16] P. Van Mieghem, Europhys. Lett. 97, 48004 (2012).

[17] A.V. Goltsev, S.N. Dorogovtsev, J.G. Oliveira, and J.F. F. Mendes, Phys. Rev. Lett. 109, 128702 (2012).

[18] M. Boguñá, C. Castellano, and R. Pastor-Satorras, Phys. Rev. Lett. 111, 068701 (2013).

[19] H. K. Lee, P.-S. Shim, and J. D. Noh, arXiv:1309.5367 (2013).

[20] H. D. Rozenfeld, S. Havlin, and D. ben-Avraham, New J. Phys. 9, 175 (2007)

[21] H. D. Rozenfeld and D. ben-Avraham, Phys. Rev. E 75, 061102 (2007).

[22] D. Stauffer and A. Aharony, Introduction to Percolation Theory (Taylor \& Francis, London, 1994).

[23] K. Christensen and N. R. Moloney, Complexity and Criticality (Imperial College Press, London, 2005).

[24] T. Hasegawa and K. Nemoto, Phys. Rev. E 88, 062807 (2013). 